\title{
Circulating tumor DNA: A promising biomarker to guide postoperative treatment and surveillance of non-small cell lung cancer
}

\author{
James M. Isbell, MD, MSCI, ${ }^{a}$ David R. Jones, MD, ${ }^{a}$ and Bob T. Li, MD ${ }^{b}$
}

From the ${ }^{\mathrm{a}}$ Thoracic Service, Department of Surgery, and ${ }^{\mathrm{b}}$ Thoracic Oncology Service, Division of Solid Tumor Oncology, Department of Medicine, Memorial Sloan Kettering Cancer Center and Weill Cornell Medical College, New York, NY.

This work was supported, in part, by the National Institutes of Health Comprehensive Cancer Center Core Grant (P30 CA008748).

Received for publication Sept 27, 2017; revisions received Nov 30, 2017; accepted for publication Dec 14, 2017; available ahead of print March 31, 2018.

Address for reprints: James M. Isbell, MD, MSCI, Thoracic Service, Department of Surgery, Memorial Sloan Kettering Cancer Center, 1275 York Ave, Box 7, New York, NY 10065 (E-mail: isbellj@mskcc.org).

J Thorac Cardiovasc Surg 2018;155:2628-31

$0022-5223 / \$ 36.00$

Copyright (c) 2018 by The American Association for Thoracic Surgery

https://doi.org/10.1016/j.jtcvs.2017.12.157

Feature Editor's Note-It has recently been discovered that non-small cell lung cancer (NSCLC) sheds measurable DNA into the circulation. The technology that can detect this circulating tumor DNA (ctDNA) is rapidly evolving and has potential to revolutionize the management of patients with both early and advanced NSCLC. Circulating tumor DNA is currently being used clinically in patients with advanced-stage NSCLC as a "liquid biopsy" to detect certain common tumor mutations that would make them candidates for targeted molecular therapy (EGFR and ALK). Within context of the field of ctDNA research, Isbell and colleagues elegantly summarize the initial results of the first 100 patients with resectable NSCLC treated in the Tracking NSCLC Evolution Through Therapy (TRACERx) multicenter clinical trial, recently published in Nature. The basic mechanics of ctDNA measurement in the clinic begin with the sampling and sequencing of the primary tumor to identify tumor-specific DNA mutations, and the peripheral blood of that individual is then interrogated for the presence of DNA containing these mutations. Tumor DNA has a short enough half-life in the circulation that it is a biomarker suitable to indicate the presence of the cancer from which it is derived, whether it be an untreated primary tumor, an incompletely resected primary, or a recurrent tumor after treatment. Thus, measurement of ctDNA may be a useful instrument for lung cancer screening and diagnosis, assessment of completeness of tumor resection, and longitudinal assessment of response to therapy. As highlighted by Isbell and colleagues, less than half of patients undergoing resection for NSCLC are alive at 5 years, and their mortality is predominantly accounted for by recurrence. Further, up to one third of patients undergoing resection of early-stage NSCLC will experience recurrence, and longitudinal ctDNA may

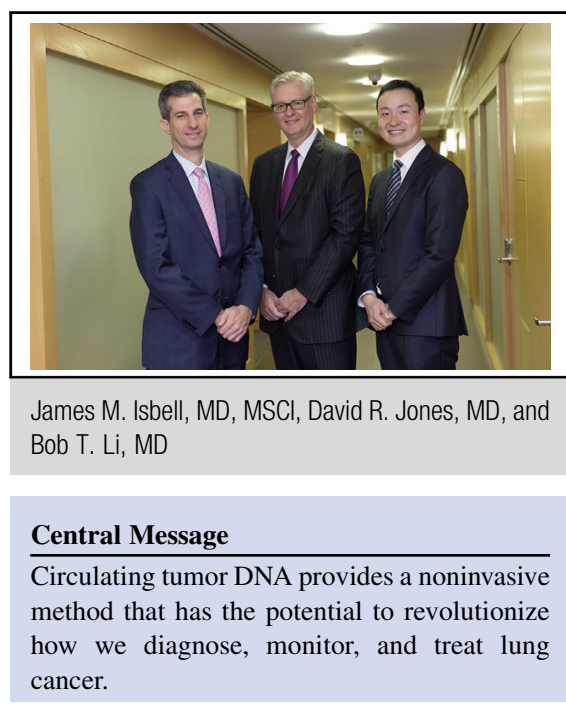

See Editorial Commentary page 2632.

improve disease monitoring for these patients. Whereas the promise of ctDNA to affect the care of patients with early-stage NSCLC is real, prospective trials are required to understand its utility for such cases, and a preview of The American Association for Thoracic Surgery-sponsored Thoracic Surgery Oncology Group ctDNA trial in resectable lung cancer is showcased by the authors of this Feature Expert Opinion article.

\section{Bryan M. Burt, MD}

Despite recent advances in systemic and radiation therapy, long-term survival after resection for non-small cell lung cancer (NSCLC) remains at less than 50\%. Cancer recurrence is the primary driver of postresection mortality. ${ }^{1}$ In addition to complete surgical resection, adjuvant chemotherapy has been the mainstay of treatment in the setting of locally advanced disease to reduce the risk of postoperative recurrence. However, according to the Lung Adjuvant Cisplatin Evaluation meta-analysis, postoperative chemotherapy provides an absolute survival benefit of only $5 \%$. $^{2}$ Patients with early-stage NSCLC experience postresectional recurrence rates as high as $24 \%$ to $36 \%{ }^{3-5}$ suggesting that the current pathologic staging methods are inadequate. Particularly lacking in our existing treatment armamentarium for NSCLC is the availability of a 
biomarker that could be used to determine whether patients are truly cancer free after presumed "complete" surgical resection. With an accurate and noninvasive method to detect minimal residual disease, exposure to the toxicities of adjuvant therapy could be limited to those patients who would most likely stand to benefit. Furthermore, using such a biomarker in the postoperative surveillance of patients may allow for earlier detection and treatment of recurrent disease, potentially resulting in improved outcomes.

To this end, several groups, including our own, are exploring the use of circulating tumor DNA (ctDNA) as a potential biomarker to indicate the presence of minimal residual or recurrent disease after surgical resection for NSCLC. Cancers typically develop through a series of sequential alterations and mutations in genes that control cell cycle and growth. These so-called somatic gene alterations, which include both genetic mutations and epigenetic changes, are present in cancer cells but not in normal cells. Although cell-free DNA is present to some extent in everyone, tumor-derived mutant DNA (ctDNA) can be found only in the circulation of patients with cancer and thus may serve as a valuable biomarker for the presence and extent of malignancy. Moreover, the relatively short half-life of circulating DNA makes it an ideal biomarker to monitor treatment response in a variety of cancers. ${ }^{6}$

In a recent issue of Nature, Abbosh and colleagues ${ }^{7}$ published a preliminary report on the phylogenetic ctDNA analysis of the first 100 patients with resectable NSCLC enrolled in the Tracking Non-Small Cell Lung Cancer Evolution Through Therapy [Rx] (TRACERx) study (NCT01888601). ${ }^{7}$ The objectives of this ongoing multicenter trial are to assess (1) the role tumor heterogeneity plays in clinical outcomes, (2) the ability of ctDNA to predict cancer relapse, and (3) the utility of ctDNA to detect actionable driver mutations identified in primary or metastatic tumors. The findings of this study, as well as those of a companion study published in The New England Journal of Medicine, ${ }^{8}$ have wide-ranging implications for patients with NSCLC and the thoracic surgeons and oncologists who care for them.

The TRACERx investigators performed multiregion sampling and whole-exome sequencing on resected NSCLC specimens. On the basis of the sequencing information obtained from the tumor specimens, patient-specific multiplex-polymerase chain reaction assay panels were synthesized. These patient-specific assay panels were then used to detect clonal and subclonal point mutations (more commonly known as "single nucleotide variants" [SNVs]) present in preoperatively and postoperatively collected ctDNA using amplicon-based next-generation sequencing techniques. The investigators set a threshold of 2 detected SNVs to specify a plasma sample as being positive for ctDNA. When this definition was used, ctDNA was detected in only 46 of the 96 preoperative early-stage NSCLC plasma samples $(48 \%)$. There was a distinct difference in preoperative detection between squamous cell carcinomas of the lung $(97 \%)$ and adenocarcinomas of the lung $(19 \%)$. This difference in detection between the 2 histologic subtypes was maintained in a stage-matched subgroup comparison of stage I squamous cell and adenocarcinoma cases. Although this may suggest that squamous cell carcinomas have a greater propensity to shed DNA into the circulation, it may also reflect technical issues with the underlying assay.

Multivariable analysis demonstrated that nonadenocarcinoma histologic subtype, lymphovascular invasion, and a high proliferative index based on Ki67 staining were independent predictors of ctDNA detection. Tumor volume, based on both pathologic and computed tomography (CT) scan volumetric analyses, correlated with ctDNA detection, as did ${ }^{18} \mathrm{~F}$-fluoro-deoxyglucose avidity on positron emission tomography. The authors estimated that a tumor volume of $10 \mathrm{~cm}^{3}$, or approximately $2.7 \mathrm{~cm}$ in diameter if spherical, would be required to shed sufficient ctDNA to be detected at the current detection thresholds of $0.1 \%$ variant allele frequency.

The longitudinal phase of the study sought to determine whether serial ctDNA collections in a subset of patients could be used to detect cancer relapse, as well as the underlying subclone(s) driving the relapse. Patients were followed up every 3 months for the first 2 years from study enrollment and then every 6 months thereafter, with a history, a physical examination, and chest radiographs. Follow-up CT scans were obtained only when warranted by clinical suspicion or concerning changes on chest radiograph. The use of chest radiographs, rather than chest CT scans, is the standard of care for postoperative surveillance in the United Kingdom, where the TRACERx study is being conducted. ctDNA was detected in 13 of 14 confirmed cases of NSCLC relapse (93\%). ctDNA detection of cancer relapse preceded CT scan detection by a median of 70 days, although this lead time would likely have been considerably reduced were the study to have been conducted using the standard postoperative surveillance CT scanning paradigm typically employed in the United States. Adjuvant chemotherapy resistance appeared to be associated with increasing numbers of postoperative SNVs detected, because the 3 patients who had increasing SNV levels while receiving postoperative chemotherapy all relapsed within 1 year. And perhaps most interestingly, the investigators were able to phylogenetically trace metastatic subclones back to the region of the primary tumor from which they likely arose.

The findings of this and other recent studies ${ }^{9-13}$ provide compelling support for the use of ctDNA as a prognostic biomarker. Although larger-scale, confirmatory studies are needed, ctDNA appears to hold promise as a tool to monitor response to therapy, as well as to detect minimal residual 
disease after surgical resection. Current adjuvant therapy guidelines for NSCLC are principally based on pathologic tumor stage. ${ }^{14}$ In stage IA NSCLC, adjuvant chemotherapy has been found to be harmful in terms of overall and disease-specific survival; in stage IB disease, its benefit remains less clear. Accordingly, the majority of patients with stage I do not currently receive postoperative chemotherapy. However, recurrence rates in stage I NSCLC are reported to be as high as $24 \%$ to $36 \%,{ }^{3-5}$ which suggests the presence of occult micrometastatic disease in a significant proportion of these "early-stage" patients. Clearly, we need a method better than the current staging system to identify patients at high risk for relapse after surgical resection.

The TRACERx investigators demonstrated not only that detectable postoperative ctDNA predicted NSCLC relapse but also that ctDNA analysis revealed the subclone(s) responsible. Likewise, on the basis of recently published preliminary studies in colorectal, breast, and lung cancer, ${ }^{12,13,15}$ the presence of persistent postoperative ctDNA appears to signal residual disease well before clinical relapse becomes evident. Moreover, patients in whom ctDNA is detected postoperatively have significantly reduced survival. ${ }^{12,13,15}$ Such high-risk patients, regardless of disease stage, could potentially benefit from postoperative systemic cytotoxic, targeted, or immune-based therapy to eradicate minimal residual disease.

Additional prospective studies are needed to validate the diagnostic, prognostic, and predictive power of ctDNA in early-stage NSCLC. The American Association for Thoracic Surgery-sponsored Thoracic Surgery Oncology Group recently agreed to support a multicenter trial to evaluate the utility of ctDNA as a prognostic and predictive biomarker in patients undergoing neoadjuvant therapy for resectable NSCLC. The neoadjuvant setting provides a unique opportunity to evaluate the effect of systemic therapy and surgical resection on ctDNA levels longitudinally within the same patient, and potentially identify new precision therapies matched to the genomic alterations in ctDNA. In addition to assessing the capabilities of preoperative and postoperative ctDNA levels to predict disease-free survival and overall survival, this study will seek to determine whether the change in ctDNA levels after neoadjuvant therapy correlates with pathologic treatment response. If validation studies confirm the utility of ctDNA as a prognostic biomarker to a high degree of sensitivity and specificity, future trials could randomize patients to current standard of care adjuvant therapy (on the basis of pathologic stage) or adjuvant therapy (on the basis of presence of ctDNA, regardless of pathologic stage). Such a ctDNAguided approach could potentially distinguish patients with micrometastatic disease from those without residual disease after resection and thereby spare the latter group of patients from the toxicities of adjuvant therapy. If a ctDNA-guided approach led to improved survival over the current stage-based approach, this could dramatically change the way we treat NSCLC in the future.

Before such ctDNA-guided approaches can be widely used clinically, several technologic barriers will need to be overcome. As is the case with most ctDNA assays, particularly in early-stage disease, the assay used in the TRACERx study requires prior knowledge of tumor sequencing results. This prerequisite for a priori knowledge stems from the fact that there is usually only a tiny fraction of ctDNA in the blood of patients with early-stage NSCLC relative to the overall amount of cell-free DNA. The TRACERx group estimates that to reach minimum ctDNA detection thresholds at $0.1 \%$ variant allele frequency with the currently available next-generation sequencing technology, a total tumor burden of $10 \mathrm{~cm}^{3}$ is required. This would be equivalent to a solitary tumor of approximately $2.7 \mathrm{~cm}$ in diameter, or a T1c tumor under the current staging system. ${ }^{16}$ Such a tumor would be easy to identify on cross-sectional imaging and would far exceed what most would qualify as minimal residual disease.

Further technologic refinements in ctDNA assays will be necessary to detect micrometastatic disease with high sensitivity after surgical resection. Additional strategies to incorporate epigenetic alterations-most notably, DNA methylation events-may improve the sensitivity and specificity of ctDNA analysis for the early detection, prognostication, and treatment surveillance of NSCLC. ${ }^{17,18}$ Pyrosequencing and droplet digital polymerase chain reaction are techniques that could be used to detect DNA methylation within the promoter regions of tumor suppressor and other cancer-specific genes. ${ }^{17}$ DNA hypermethylation of such promoter sequences occurs early in carcinogenesis, permanently disabling expression of the corresponding gene. In NSCLC, DNA hypermethylation is associated with disease progression and poor survival. ${ }^{17}$ Of note, DNA methylation is detectable in plasma ctDNA, which enhances the usefulness of ctDNA as a biomarker.

Some investigators have recently reported encouraging results with de novo detection of ctDNA sequence alterations at relatively low thresholds, without the need for prior knowledge of genetic tumor sequencing. ${ }^{10,13}$ Nonetheless, this remains a developing technology and one that needs further evaluation and validation before it can be used in the detection of minimal residual disease after surgical resection. As previously mentioned, another issue highlighted in the TRACERx study is the widely divergent rates of preoperative ctDNA detection between lung squamous cell carcinoma and lung adenocarcinoma $(97 \%$ vs $19 \% ; P=.001)$. Although there may be true biological differences between histologies in their tumor DNA shedding, this stark difference in detection has not been reported by other groups who have studied ctDNA in early-stage lung cancers, ${ }^{9,10,13}$ and thus it is plausible that technologic factors related to the assay used, as well as the 
higher proportion of patients with stage I in the TRACERx study, might have also contributed to this difference.

Although considerable technologic advances still need to be made, ctDNA promises to become a valuable tool in the early detection and management of NSCLC. Used as an adjunct to low-dose chest CT screening, which had a $96.4 \%$ false-positive rate in the landmark National Lung Screening Trial, ${ }^{19}$ ctDNA may eventually play a role in reducing the number of unnecessary diagnostic procedures associated with screening. In the future, one could envision a complete (R0) resection being redefined as a nondetectable postoperative ctDNA level. Likewise, ctDNA could be used in the surveillance algorithm of patients previously treated with stereotactic body radiotherapy. Given the exponential increase in the use of stereotactic body radiotherapy to treat early-stage NSCLC, ${ }^{20}$ appropriate patients with persistently detectable ctDNA post-treatment could be offered surgical resection. In the meantime, ctDNA is currently being used clinically for "liquid biopsies" in the metastatic setting as a noninvasive means of determining whether patients' tumors harbor known activating gene alterations, such as $A L K$ and $E G F R$, which would make them candidates for targeted therapy.

\section{CONCLUSIONS}

ctDNA has the potential to revolutionize how we diagnose, monitor, and treat cancer. Several characteristics make ctDNA an ideal biomarker, including its short half-life, its direct linkage to the presence of malignancy, and its noninvasive means of access. Although ctDNA as a diagnostic and prognostic tool remains in its infancy, advancements in the underlying technology are developing rapidly and will undoubtedly expand its clinical applicability in the near future. Earlier identification of minimal residual disease or recurrence through ctDNA surveillance could result in more timely treatment and improved survival. Before reaching this point, however, well-designed clinical trials must be conducted to prove the efficacy of this promising technology.

\section{Conflict of Interest Statement}

B.T.L. has served as a consultant for ThermoFisher Scientific and Genentech and has received research funding from Grail. All other authors have nothing to disclose with regard to commercial support.

The authors thank David Sewell for the editorial assistance in preparing this manuscript.

\section{References}

1. Eguchi T, Bains S, Lee MC, Tan KS, Hristov B, Buitrago DH, et al. Impact of increasing age on cause-specific mortality and morbidity in patients with stage I non-small-cell lung cancer: a competing risks analysis. J Clin Oncol. 2017; 35:281-90.

2. Pignon JP, Tribodet H, Scagliotti GV, Douillard JY, Shepherd FA, Stephens RJ, et al. Lung adjuvant cisplatin evaluation: a pooled analysis by the LACE Collaborative Group. J Clin Oncol. 2008;26:3552-9.

3. Goodgame B, Viswanathan A, Zoole J, Gao F, Miller CR, Subramanian J, et al. Risk of recurrence of resected stage I non-small cell lung cancer in elderly patients as compared with younger patients. J Thorac Oncol. 2009; 4:1370-4.

4. Taylor MD, Nagji AS, Bhamidipati CM, Theodosakis N, Kozower BD, Lau CL, et al. Tumor recurrence after complete resection for non-small cell lung cancer. Ann Thorac Surg. 2012;93:1813-20.

5. Kelsey CR, Marks LB, Hollis D, Hubbs JL, Ready NE, D’Amico TA, et al. Local recurrence after surgery for early stage lung cancer: an 11-year experience with 975 patients. Cancer. 2009; 115:5218-27.

6. Lo YM, Zhang J, Leung TN, Lau TK, Chang AM, Hjelm NM. Rapid clearance of fetal DNA from maternal plasma. Am J Hum Genet. 1999; 64:218-24.

7. Abbosh C, Birkbak NJ, Wilson GA, Jamal-Hanjani M, Constantin T, Salari R, et al. Phylogenetic ctDNA analysis depicts early-stage lung cancer evolution. Nature. 2017;545:446-51.

8. Jamal-Hanjani M, Wilson GA, McGranahan N, Birkbak NJ, Watkins TBK, Veeriah S, et al. Tracking the evolution of non-small-cell lung cancer. $N$ Engl J Med. 2017;376:2109-21.

9. Newman AM, Bratman SV, To J, Wynne JF, Eclov NC, Modlin LA, et al. An ultrasensitive method for quantitating circulating tumor DNA with broad patient coverage. Nat Med. 2014;20:548-54.

10. Phallen J, Sausen M, Adleff V, Leal A, Hruban C, White J, et al. Direc detection of early-stage cancers using circulating tumor DNA. Sci Transl Med. 2017;9.

11. Diehl F, Schmidt K, Choti MA, Romans K, Goodman S, Li M, et al. Circulating mutant DNA to assess tumor dynamics. Nat Med. 2008;14: 985-90.

12. Tie J, Wang Y, Tomasetti C, Li L, Springer S, Kinde I, et al. Circulating tumor DNA analysis detects minimal residual disease and predicts recurrence in patients with stage II colon cancer. Sci Transl Med. 2016; 8:346ra92.

13. Chaudhuri AA, Chabon JJ, Lovejoy AF, Newman AM, Stehr H, Azad TD, et al. Early detection of molecular residual disease in localized lung cancer by circulating tumor DNA profiling. Cancer Discov. 2017;7:1394-403.

14. Kris MG, Gaspar LE, Chaft JE, Kennedy EB, Azzoli CG, Ellis PM, et al Adjuvant systemic therapy and adjuvant radiation therapy for stage I to IIIA completely resected non-small-cell lung cancers: American Society of Clinical Oncology/cancer care Ontario clinical practice guideline update. J Clin Oncol. 2017;35:2960-74.

15. Olsson E, Winter C, George A, Chen Y, Howlin J, Tang MH, et al Serial monitoring of circulating tumor DNA in patients with primary breast cancer for detection of occult metastatic disease. EMBO Mol Med. 2015;7: 1034-47.

16. American Joint Committee on Cancer. AJCC Cancer Staging Manual. 8th ed. New York, NY: Springer; 2016.

17. Lissa D, Robles AI. Methylation analyses in liquid biopsy. Transl Lung Cancer Res. 2016;5:492-504.

18. Sandoval J, Mendez-Gonzalez J, Nadal E, Chen G, Carmona FJ, Sayols S, et al. A prognostic DNA methylation signature for stage I non-small-cell lung cancer. J Clin Oncol. 2013;31:4140-7.

19. National Lung Screening Trial Research Team, Aberle DR, Adams AM, et al Reduced lung-cancer mortality with low-dose computed tomographic screening. N Engl J Med. 2011;365:395-409.

20. McMurry TL, Shah PM, Samson P, Robinson CG, Kozower BD. Treatment of stage I non-small cell lung cancer: what's trending? J Thorac Cardiovasc Surg. 2017;154:1080-7. 